\title{
COMPACTNESS CRITERIA IN WEIGHTED VARIABLE LEBESGUE SPACES
}

\author{
ROVSHAN A. BANDALIYEV
}

Received 18 October, 2015

\begin{abstract}
In this paper a compactness criterion in weighted variable Lebesgue spaces is proved. In particular, are proved a compactness criterion in variable exponent sequence Lebesgue spaces.

2010 Mathematics Subject Classification: 46B50; 46E30; 28C99

Keywords: weighted variable Lebesgue spaces, variable exponent sequence Lebesgue spaces, compactness, convolution type operator, maximal function
\end{abstract}

\section{INTRODUCTION}

Compactness results in the usually Lebesgue spaces are often vital in existence proofs for nonlinear partial differential equations. A necessary and sufficient condition for a subset of usually Lebesgue spaces to be compact is given in what is often called the Kolmogorov compactness theorem, or Frechet-Kolmogorov theorem. Furthemore, we trace out the historical roots of Kolmogorov compactness theorem, which originated in [12] (see also [19]).

In this paper we extend Kolmogorov compactness criterion to the case of weighted variable Lebesgue spaces. The aim of this paper is to give a characterization of precompact sets in weighted variable Lebesgue space. Moreover, we study the precompactness of weighted sequence spaces. The theory of variable Lebesgue spaces was intensively developed during the last two decades, inspired both by difficult open problems in this theory and possibly applications shown in [16]. We refer to detail in $[1-4,13,17,18]$ and e.t.c.

Let us mention some generalizations of the Riesz-Kolmogorov theorem. Recently in [7] and [11] was shown the Riesz-Kolmogorov compactness theorem on metric spaces. In [8] full characterization of relatively compact sets is given in the case of variable Lebesgue spaces on metric measure spaces. In [20] the compactness theorem

This work was supported by the grant of Science Development Foundation under the President of the Republic of Azerbaijan, Grant EIF-2013-9(15)-46/10/1 and by the grant of Presidium of Azerbaijan National Academy of Science 2015. 
in $L^{p}(G)$ on locally compact group $G$ is shown. In [14] Kolmogorov theorem for $p=2$ in terms of the Fourier transform is given (see also [5, 6]).

\section{PReliminaries}

An $\varepsilon$-cover of a metric space is a cover of the space consisting of sets of diameter at most $\varepsilon$. A metric space is called totally bounded if it admits a finite $\varepsilon$-cover for all $\varepsilon$. It is well known that a metric space is compact if and only if it is complete and totally bounded (see [21]). Since we are interested in compactness results for subsets of Banach spaces, we concentrate our attention on total boundedness.

Let $R^{n}$ be the $n$-dimensional Euclidean space of points $x=\left(x_{1}, \ldots, x_{n}\right)$. Let $p$ be a Lebesgue measurable function on $R^{n}$ such that $1 \leq p \leq p(x) \leq \bar{p} \leq \infty, p=$ ess $\inf _{x \in R^{n}} p(x), \bar{p}=e s s \sup _{x \in R^{n}} p(x)$, and $\omega$ is a weight function on $R^{n}$, i.e. $\omega$ is non-negative, almost everywhere (a.e.) positive function on $R^{n}$. The Lebesgue measure of a set $\Omega$ will be denoted by $|\Omega|$.

Definition 1. By $L_{p(x), \omega}\left(R^{n}\right)$ we denote the set of all measurable functions $f$ on $R^{n}$ such that for some $\lambda_{0}>0$

$$
I_{p, \omega}(f)=\int_{R^{n}}\left(\frac{|f(x)|}{\lambda_{0}}\right)^{p(x)} \omega(x) d x<\infty .
$$

Note that the expression

$$
\|f\|_{L_{p(\cdot), \omega}\left(R^{n}\right)}=\|f\|_{p(\cdot), \omega}=\inf \left\{\lambda>0: \int_{R^{n}}\left(\frac{|f(x)|}{\lambda}\right)^{p(x)} \omega(x) d x \leq 1\right\}
$$

defines the norm in the space $L_{p(x), \omega}\left(R^{n}\right)$. The spaces $L_{p(x), \omega}\left(R^{n}\right)$ is a Banach function space with respect to the norm (see [3]).

By $P^{\log }\left(R^{n}\right)$ we denote the class of variable exponents satisfying following condition:

$$
\begin{gathered}
|p(x)-p(y)| \leq \frac{C_{1}}{-\ln |x-y|}, \quad 0<|x-y| \leq \frac{1}{2}, \\
\left|p(x)-p_{\infty}\right| \leq \frac{C_{2}}{\ln (e+|x|)}, \quad|x|>2,
\end{gathered}
$$

where $C_{1}$ and $C_{2}$ are positive constants independents on $x, y$ and $\lim _{|y| \rightarrow \infty} p(y)=p_{\infty}$.

Let us define the class $A_{p(\cdot)}$ consisting of those weights $\omega \in L_{1}^{l o c}\left(R^{n}\right)$ such that

$$
\sup _{B}|B|^{-1}\left\|\omega^{1 / p(\cdot)}\right\|_{L_{p(\cdot)}}\left\|\omega^{-1 / p(\cdot)}\right\|_{L_{p^{\prime}(\cdot)}}<\infty,
$$

where the supremum is taken over all balls $B \subset R^{n}$ and $\frac{1}{p(x)}+\frac{1}{p^{\prime}(x)}=1$. 
Here is the key lemma for many compactness results.

Lemma 1. [9] Let $X$ be a metric space. Assume that, for every $\varepsilon>0$, there exists some $\delta>0$, a metric space $W$, and a mapping $\Phi: X \mapsto W$ so that $\Phi[X]$ is totally bounded, and whenever $x, y \in X$ are such that $d(\Phi(x), \Phi(y))<\delta$, then $d(x, y)<\varepsilon$. Then $X$ is totally bounded.

Let $f \in L_{1}^{l o c}\left(R^{n}\right)$ and we define the Hardy-Littlewood maximal function

$$
M f(x)=\sup _{x \in B}|B|^{-1} \int_{B}|f(y)| d y
$$

and

$$
(f * g)(x)=\int_{R^{n}} f(y) g(x-y) d y
$$

denoted the usual convolution operator.

Theorem 1. [10] Let $p \in P^{\log }\left(R^{n}\right)$ with $1<p \leq \bar{p}<\infty$ and $\omega$ is a weight function.

Then $M: L_{p(x), \omega}\left(R^{n}\right) \mapsto L_{p(x), \omega}\left(R^{n}\right)$ is bounded if and only if $\omega \in A_{p(\cdot)}$. The embedding constant depends on $p$ and $\omega$.

Theorem 2. Let $p \in P^{\log }\left(R^{n}\right)$ and let $\psi: R^{n} \mapsto R$ be an integrable function. Assume that $\psi_{\varepsilon}(x):=\varepsilon^{-n} \psi\left(\frac{x}{\varepsilon}\right)$ for all $\varepsilon>0$ and $\Psi(x):=\sup _{|y| \geq|x|} \psi(y) \in L_{1}\left(R^{n}\right)$. Then

a) Then exists $A>0$ such that $\sup _{\varepsilon>0}\left|\left(f * \psi_{\varepsilon}\right)(x)\right| \leq 2 A M f(x)$, where A depends on $\Psi, f \in L_{p(x), \omega}\left(R^{n}\right)$ and $M$ is Hardy-Littlewood maximal operator;

b) If $\int_{R^{n}} \psi(x) d x=1$, then for $f \in L_{p(x), \omega}\left(R^{n}\right)$ we have $f * \psi_{\varepsilon} \rightarrow f$ for $\varepsilon \rightarrow 0$ a. e. $x \in R^{n}$. If additionally $\bar{p}<\infty$, then $\lim _{\varepsilon \rightarrow 0+}\left\|f * \psi_{\varepsilon}-f\right\|_{p(\cdot), \omega}=0$.

Proof of Theorem 2 analogously to the proof of non-weighted case (see [4]). Thus, from Theorem 1 and Theorem 2 (a) we obtain the following

Theorem 3. Let $p \in P^{\log }\left(R^{n}\right)$ with $1<\underline{p} \leq \bar{p}<\infty$ and $\omega \in A_{p(\cdot)}$. Then

$$
\left\|f * \psi_{\varepsilon}\right\|_{p(\cdot), \omega} \leq 2 A\|f\|_{p(\cdot), \omega},
$$

where $A$ is independent of $\varepsilon$ and $f$. 


\section{MAin RESUlts.}

Let $w=\left\{w_{n}\right\}$ be a sequence of positive numbers. Let

$$
l_{p_{n}}(w):=\left\{x: x=\left(x_{1}, \ldots, x_{n}, \ldots\right), \sum_{k=1}^{\infty}\left(\frac{\left|x_{k}\right|}{\lambda_{0}}\right)^{p_{k}} w_{k}<\infty\right\}
$$

denote weighted variable sequence Lebesgue spaces with the norm

$$
\|x\|_{l_{p_{n}}(w)}:=\left\|x w^{\frac{1}{p_{n}}}\right\|_{l_{p_{n}}}=\inf \left\{\lambda>0: \sum_{k=1}^{\infty}\left(\frac{\left|x_{k}\right|}{\lambda}\right)^{p_{k}} w_{k} \leq 1\right\} .
$$

Theorem 4. Let $1 \leq p_{n} \leq \sup _{n} p_{n}<\infty, w=\left\{w_{n}\right\}$ be a sequence of positive numbers and $S \subset l_{p_{n}}(w)$. A subset $S$ is totally bounded if and only if:

(i) it is pointwise bounded;

(ii) for every $\varepsilon>0$ there is some $n$ such that, for every $x \in S$ $\left\|x w^{\frac{1}{p_{k}}}\right\|_{l_{p_{k}}(k>n)}<\varepsilon$.

Proof of Theorem 4. Let $S \subset l_{p_{n}}(w)$ satisfies the two condition. Given $\varepsilon>0$, fix $n$ as in the second condition, and define a mapping $\Phi: S \mapsto R^{n}$ by

$$
\Phi(x)=\left(x_{1}, \ldots, x_{n}\right) .
$$

By the pointwise boundedness of $S$, the image $\Phi(S)$ is totally bounded. Let $x, y \in S$ with

$$
|\Phi(x)-\Phi(y)|_{p_{n}(w)}=\inf \left\{\lambda>0: \sum_{k \leq n}\left(\frac{\left|x_{k}-y_{k}\right|}{\lambda}\right)^{p_{k}} w_{k} \leq 1\right\}<\varepsilon .
$$

We have

$$
\begin{aligned}
& \|x-y\|_{l_{p_{n}}(w)} \\
& \leq 2\left(|\Phi(x)-\Phi(y)|_{l_{p_{n}}(w)}+\inf \left\{\lambda>0: \sum_{k=n+1}^{\infty}\left(\frac{\left|x_{k}-y_{k}\right|}{\lambda}\right)^{p_{k}} w_{k} \leq 1\right\}\right) \\
& <2 \varepsilon+2 \varepsilon=4 \varepsilon .
\end{aligned}
$$

By Lemma $1, \mathrm{~S}$ is totally bounded.

Note that conditions $(i)$ and $(i i)$ are also necessary.

Remark 1. Note that in the case $w=1$ Theorem 4 was proved in [8]. Also, for $p(x)=p=$ const,$\omega=1$ Theorem 4 was proved in [9]. 
Theorem 5. Let $p \in P^{\log }\left(R^{n}\right)$ and $1 \leq p \leq p(x) \leq \bar{p}<\infty$. Suppose that $\omega$ is a weight function on $R^{n}$ and $\omega \in A_{p(\cdot)}$. Then a subset $S$ of $L_{p(x), \omega}\left(R^{n}\right)$ is totally bounded if and only if:

1) $S$ is bounded in $L_{p(x), \omega}\left(R^{n}\right)$, i.e. $\sup _{f \in S}\|f\|_{p(\cdot), \omega}<\infty$;

2) for every $\varepsilon>0$ there is some $\eta>0$ such that, for every $f \in S\|f\|_{L_{p(\cdot), \omega}(|x|>\eta)}<$ $\varepsilon$

3) $\lim _{\varepsilon \rightarrow 0+}\left\|f * \psi_{\varepsilon}-f\right\|_{p(\cdot), \omega}=0$, uniformly for $f \in S$.

Proof of Theorem 5. Necessity. Assume that $S$ is totally bounded. The existence of a finite $\varepsilon$-cover for $S$, for any $\varepsilon$, clearly implies the boundedness of $S$, thus establishing condition 1 ).

Now we prove the necessity of 2). To establish condition 2), let $\varepsilon>0$ be given and let $\left\{U_{1}, \ldots, U_{m}\right\}$ be an $\varepsilon$-cover of $S$ and choose $g_{j}$ for $j=1, \ldots, m$. Select $\eta>0$ such that

$$
\left\|g_{j}\right\|_{L_{p(\cdot), \omega}(|x|>\eta)}<\varepsilon, \quad j=1, \ldots, m .
$$

If $f \in U_{j}$, then $\left\|f-g_{j}\right\|_{p(\cdot), \omega} \leq \varepsilon$, and so

$$
\|f\|_{L_{p(\cdot), \omega}(|x|>\eta)} \leq\left\|f-g_{j}\right\|_{L_{p(\cdot), \omega}(|x|>\eta)}+\left\|g_{j}\right\|_{L_{p(\cdot), \omega}(|x|>\eta)} \leq 2 \varepsilon,
$$

thus establishing condition 2).

To prove 3 ) we first note that, by Theorem $2 \mathrm{~b}$ ), given $\varepsilon>0$, there exists an $h_{k}$ indexed to each $g_{k}$ such that

$$
\left\|\psi_{h} * g_{k}-g_{k}\right\|_{p(\cdot), \omega}<\varepsilon
$$

where $h<h_{k}$. Put $h_{0}=\min _{1 \leq k \leq l} h_{k}$, we have $\left\|\psi_{h} * g_{k}-g_{k}\right\|_{p(\cdot), \omega}<\varepsilon$ for all $k=$ $1, \ldots, l$ where $h<h_{0}$. Then for $h<h_{0}$ and all $f \in S$ by Theorem 3 we have a suitable $g_{r}$ such that

$$
\begin{gathered}
\left\|\psi_{h} * f-f\right\|_{p(\cdot), \omega} \leq\left\|\psi_{h} *\left(f-g_{r}\right)\right\|_{p(\cdot), \omega}+\left\|f-g_{r}\right\|_{p(\cdot), \omega}+\left\|\psi_{h} * g_{r}-g_{r}\right\|_{p(\cdot), \omega} \\
\leq(2 A+1)\left\|f-g_{r}\right\|_{p(\cdot), \omega}+\varepsilon<(2 A+2) \varepsilon,
\end{gathered}
$$

which proves the necessity of 3 ).

Sufficiency part of Theorem 5 is proved analogously to the non-weighted case, i.e. when $\omega=1$ (see [15]).

This completes the proof of Theorem 5.

Remark 2. Note that in the case $\omega=1$ and when $\Omega \subset R^{n}$ is a bounded open set Theorem 5 was proved in [15]. Also, for $p(x)=p=$ const, $\omega=1$ and other condition than 3) Theorem 5 was proved in [9]. 


\section{ACKNOWLEDGEMENT}

The author would like to thank the referee for his/her valuable comments and suggestions which helped to improve the manuscript.

\section{REFERENCES}

[1] R. A. Bandaliev, "On an inequality in a Lebesgue space with mixed norm and variable summability exponent," Mat. Zametki, vol. 84, no. 3, pp. 323-333, 2008, doi: 10.1134/S0001434608090010. [Online]. Available: http://dx.doi.org/10.1134/S0001434608090010

[2] R. A. Bandaliev, "Application of multidimensional Hardy operator and its connection with a certain nonlinear differential equation in weighted variable Lebesgue spaces," Ann. Funct. Anal., vol. 4, no. 2, pp. 118-130, 2013, doi: 10.15352/afa/1399899530. [Online]. Available: http://dx.doi.org/10.15352/afa/1399899530

[3] D. V. Cruz-Uribe and A. Fiorenza, Variable Lebesgue spaces, ser. Applied and Numerical Harmonic Analysis. Birkhäuser/Springer, Heidelberg, 2013, foundations and harmonic analysis. [Online]. Available: http://dx.doi.org/10.1007/978-3-0348-0548-3. doi: 10.1007/978-3-03480548-3

[4] L. Diening, P. Harjulehto, P. Hästö, and M. Růžička, Lebesgue and Sobolev spaces with variable exponents, ser. Lecture Notes in Mathematics. Springer, Heidelberg, 2011, vol. 2017. [Online]. Available: http://dx.doi.org/10.1007/978-3-642-18363-8. doi: 10.1007/978-3-642-18363-8

[5] P. Górka, "Pego theorem on locally compact abelian groups," J. Algebra Appl., vol. 13, no. 4, pp. 1350143,5 , 2014, doi: 10.1142/S0219498813501430. [Online]. Available: http://dx.doi.org/10.1142/S0219498813501430

[6] P. Górka and T. Kostrzewa, "Pego everywhere," J. Algebra Appl., vol. 15, no. 4, pp. 1650074,3 , 2016, doi: 10.1142/S0219498816500742. [Online]. Available: http: //dx.doi.org/10.1142/S0219498816500742

[7] P. Górka and A. Macios, "The Riesz-Kolmogorov theorem on metric spaces," Miskolc Math. Notes, vol. 15, no. 2, pp. 459-465, 2014.

[8] P. Górka and A. Macios, "Almost everything you need to know about relatively compact sets in variable Lebesgue spaces," J. Funct. Anal., vol. 269, no. 7, pp. 1925-1949, 2015, doi: 10.1016/j.jfa.2015.06.024. [Online]. Available: http://dx.doi.org/10.1016/j.jfa.2015.06.024

[9] H. Hanche-Olsen and H. Holden, "The Kolmogorov-Riesz compactness theorem," Expo. Math., vol. 28, no. 4, pp. 385-394, 2010, doi: 10.1016/j.exmath.2010.03.001. [Online]. Available: http://dx.doi.org/10.1016/j.exmath.2010.03.001

[10] P. Hästö and L. Diening, Muckenhoupt weights in variable Lebesgue spaces, ser. Preprint. Albert Ludwings Universität Freiburg, Mathematische Fakultat. [Online]. Available: http://www.helsinki.fi/pharjule/varsob/publications.shtml

[11] A. Kałamajska, "On compactness of embedding for Sobolev spaces defined on metric spaces," Ann. Acad. Sci. Fenn. Math., vol. 24, no. 1, pp. 123-132, 1999.

[12] A. Kolmogorov, "Über die kompaktheit der funktionenmengen bei der konvergenz im mittle," Nachr. Ges. Wiss. G"ottingen, vol. 9, pp. 60-63, 1931.

[13] W. Orlicz, "Über konjugierte exponentenfolgen," Stud. Math., vol. 3, pp. 200-211, 1931.

[14] R. L. Pego, "Compactness in $L^{2}$ and the Fourier transform," Proc. Amer. Math. Soc., vol. 95, no. 2, pp. 252-254, 1985, doi: 10.2307/2044522. [Online]. Available: http://dx.doi.org/10.2307/2044522

[15] H. Rafeiro, "Kolmogorov compactness criterion in variable exponent Lebesgue spaces," Proc. A. Razmadze Math. Inst., vol. 150, pp. 105-113, 2009. 
[16] M. Růžička, Electrorheological fluids: modeling and mathematical theory, ser. Lecture Notes in Mathematics. Springer-Verlag, Berlin, 2000, vol. 1748. [Online]. Available: http://dx.doi.org/10.1007/BFb0104029. doi: 10.1007/BFb0104029

[17] S. Samko, "On a progress in the theory of Lebesgue spaces with variable exponent: maximal and singular operators," Integral Transforms Spec. Funct., vol. 16, no. 5-6, pp. 461-482, 2005, doi: 10.1080/10652460412331320322. [Online]. Available: http: //dx.doi.org/10.1080/10652460412331320322

[18] I. I. Sharapudinov, “The topology of the space $\mathscr{L}^{p(t)}([0,1]), "$ Mat. Zametki, vol. 26, no. 4, pp. 613-632, 655, 1979

[19] V. N. Sudakov, "Criteria of compactness in function spaces," Uspehi Mat. Nauk (N.S.), vol. 12, no. 3(75), pp. 221-224, 1957.

[20] A. Weil, L'int'ergation dans les groupes topologiques et ses applications, 2nd ed., ser. Publications de l'Institut de matheématique de l'Universiteé de Strasbourg, 4. [Actualiteés scientifiques et industrielles, 869-1145]. Paris, Hermann, vol. 123.

[21] K. Yosida, Functional analysis, 6th ed., ser. Grundlehren der Mathematischen Wissenschaften [Fundamental Principles of Mathematical Sciences]. Springer-Verlag, Berlin-New York, vol. 123.

Author's address

Rovshan A. Bandaliyev

Institute of Mathematics and Mechanics, National Academy of Sciences of Azerbaijan, 9 B.Vahabzade

Str., AZ1141, 01141 Baku, Azerbaijan

E-mail address: bandaliyev.rovshan@math.ab.az 